\title{
The photometric evolution of dissolving star clusters
}

\section{First predictions}

\author{
H. J. G. L. M. Lamers ${ }^{1,2}$, P. Anders ${ }^{3}$, and R. de Grijs ${ }^{4}$ \\ 1 Astronomical Institute, Utrecht University, Princetonplein 5, 3584CC Utrecht, The Netherlands \\ e-mail: lamers@astro.uu.nl \\ 2 SRON Laboratory for Space Research, Sorbonnelaan 2, 3584CC, Utrecht, The Netherlands \\ 3 Institut für Astrophysik, Georg-August-Universität, Friedrich-Hund-Platz 1, 37077 Göttingen, Germany \\ e-mail: panders@astro.physik.uni-goettingen.de \\ ${ }^{4}$ Department of Physics \& Astronomy, University of Sheffield, Hicks Building, Hounsfield Road, Sheffield S3 7RH, UK \\ e-mail: R.deGrijs@sheffield.ac.uk
}

Received 5 January 2006 / Accepted 25 January 2006

\section{ABSTRACT}

\begin{abstract}
The broad-band photometric evolution of unresolved star clusters is calculated in a simplified way, including the preferential loss of low-mass stars due to mass segregation. The stellar mass function of a cluster evolves due to three effects: (a) the evolution of the massive stars reduces their number; (b) tidal effects before cluster-wide mass segregation reduce the mass function homogeneously, i.e. independently of the stellar mass; (c) after mass segregation has finished, tidal effects preferentially remove the lowest-mass stars from the cluster. These effects result in a narrowing of the stellar mass range. These effects are described quantitatively, following the results of $N$-body simulations, and are taken into account in the calculation of the photometric history, based on the GALEV cluster evolution models for solar metallicity and a Salpeter mass function. We find the following results:

(1) During the first $\sim 40 \%$ of the lifetime of a cluster its colour evolution is adequately described by the standard GALEV models (without mass segregation) but the cluster becomes fainter due to the loss of stars by tidal effects.

(2) Between $\sim 40$ and $\sim 80 \%$ of its lifetime (independent of the total lifetime), the cluster becomes bluer due to the loss of low-mass stars. This will result in an underestimate of the age of clusters if standard cluster evolution models are used.

(3) After $\sim 80 \%$ of the total lifetime of a cluster it will rapidly become redder. This will result in an overestimate of the age of clusters if standard cluster evolution models are used.

(4) Clusters with mass segregation and the preferential loss of low-mass stars evolve along almost the same tracks in colour-colour diagrams as clusters without mass segregation. Only if the total lifetime of clusters can be estimated can the colours be used to give reliable age estimates.

(5) The changes in the colour evolution of unresolved clusters due to the preferential loss of low-mass stars will affect the determination of the star formation histories of galaxies if they are derived from clusters that have a total lifetime of less than about 30 Gyr.

(6) The preferential loss of low-mass stars might explain the presence of old ( 13 Gyr) clusters in NGC 4365 which are photometrically disguised as intermediate-age clusters (2-5 Gyr), if the expected total lifetime of these clusters is between 16 and 32 Gyr.
\end{abstract}

Key words. Galaxy: open clusters and associations: general - Galaxy: globular clusters: general - galaxies: stellar content galaxies: star clusters - galaxies: startburst - galaxies: individual: NGC 4365

\section{Introduction}

The preferential loss of low-mass stars due to mass segregation in star clusters is not taken into account in the cluster photometry models available in the literature. However, this effect is of importance for the photometric evolution of "simple" stellar population models (e.g. Leitherer et al. 1999; Bruzual \& Charlot 1993; Anders \& Fritze-v. Alvensleben 2003) and hence the luminosity and mass functions of cluster populations that are derived from the analysis of observed spectral energy distributions (SEDs) of unresolved clusters compared to standard models. In this paper we describe the expected effect of this preferential loss of low-mass stars during the evolution of dissolving clusters, using a simplified description for the dynamical mass-loss mechanism. We compare and confirm this approach based on the results of $N$-body simulations.

$N$-body simulations of clusters in tidal fields show that the massive stars tend to concentrate towards the cluster center and the low-mass stars preferentially populate the outer regions. This "mass segregation" is commonly observed in open and globular clusters in the Milky Way and in the Magellanic Clouds (e.g., de Grijs et al. 2002a,b,c, and references therein).

An immediate and important consequence of mass segregation is that low-mass stars tend to be more easily, and predominantly, ejected from star clusters than high-mass stars (but see Brandl et al. 2001; de Grijs et al. 2002a). Baumgardt \& Makino (2003, hereafter BM2003) calculated the changes in the MF of clusters in the tidal field of the Galaxy. They showed that the amplitude and shape of the MF initially decrease almost homologously: the mass distribution of the stars that survive stellar evolution decreases with almost constant shape up to some critical time, after which the mass distribution of the low-mass stars becomes very steep due to the preferential loss of the lowest-mass stars. Their $N$-body simulations of clusters at different galactocentric distances in the Galaxy and with different orbits (circular vs. eccentric) show that mass segregation 
becomes important when a cluster has lost a certain fraction of its mass (approximately 70\%), almost independent of the dissolution time-scale. In these simulations the role of binaries is not taken into account, although binaries are important for mass segregation (e.g. Inagaki \& Saslaw 1985; Bonnell \& Davies 1998; Portegies Zwart et al. 1999). Therefore, in real clusters mass segregation might occur earlier than predicted by the $N$-body simulations of BM2003. In some clusters mass segregation might even be established from the beginning (e.g. Hillenbrand 1997). We will refer to this as "primordial mass segregation".

In this paper we describe the expected effect of the preferential loss of low-mass stars, due to mass segeration, on the integrated photometric evolution of clusters. We use a simplified method that enables us to explain and predict the effects for clusters of widely different lifetimes (e.g. as derived by Lamers et al. 2005a).

The structure of this paper is as follows. In Sect. 2 we provide an overview of the observational evidence for primordial and dynamical mass segregation. We discuss the assumptions of our cluster models in Sect. 3, i.e., we give a description of the decreasing mass of a cluster due to the combination of stellar evolution and evaporation or dissolution. In Sect. 4 we describe the way in which we treat the loss of stars from the cluster due to stellar evolution and dissolution. In Sect. 5 we predict the photometric evolution of clusters, taking the preferential loss of low-mass stars into account. We compare the results with those of the standard GALEV simple stellar population models (Schulz et al. 2002; Anders \& Fritze-v.-Alvernsleben 2003) and describe the expected errors in the age determinations of unresolved clusters. Section 6 contains a discussion and a suggestion of a possible explanation for the presence of an apparent intermediate age cluster population in the giant elliptical galaxy NGC 4365. The conclusions are given in Sect. 7.

\section{Observational evidence for mass segregation}

In the standard picture, stars in dense clusters evolve rapidly towards a state of energy equipartition through stellar encounters, with corresponding mass segregation. The time-scale on which dynamical mass segregation on cluster-wide scales is believed to occur is the half-mass relaxation time-scale (e.g., Inagaki \& Saslaw 1985). However, observations of various degrees of mass segregation in very young Galactic star clusters (e.g., Hillenbrand 1997; Testi et al. 1997; Fischer et al. 1998; Hillenbrand \& Hartmann 1998; Hillenbrand \& Carpenter 2000) suggest that at least some of this effect is related to the process of star and star cluster formation itself (i.e. primordial mass segregation): these clusters are often significantly younger than their two-body relaxation time (even the equivalent relaxation time in the core).

The same effect is found in several Magellanic Cloud clusters. Ground-based studies of several rich compact LMC clusters, e.g. NGC 2100 (Westerlund 1961); NGC 2098 and SL 666 (Kontizas et al. 1998), show strong indications of mass segregation. In addition, observations with the HST have also resulted in convincing cases for mass segregation in Magellanic Cloud star clusters: e.g., Fischer et al. (1998); Elson et al. (1999); Santiago et al. (2001); de Grijs et al. (2002a,b,c), Sirianni et al. (2002) and Gouliermis et al. (2004).

The clearest evidence for primordial mass segregation is found in the Orion Nebula Cluster (ONC), NGC 3603 and R136. Hillenbrand (1997) and Hillenbrand \& Hartmann (1998) presented clear evidence for mass segregation in the ONC for the $m>5 M_{\odot}$ component, with some evidence for general mass segregation down to $m \simeq 1-2 M_{\odot}$ (see the review by Larson 1993). Ground and HST-based observations of NGC 3603, one of the few moderately massive young star clusters in the Galaxy, have shown strong evidence for mass segregation in this very young ( $1 \pm 1$ Myr-old) cluster (Nürnberger \& Petr-Gotzens 2002; Stolte et al. 2004; Sung \& Bessell 2004; Grebel 2004). The cluster R136 in the 30 Doradus star-forming region in the LMC (age §3-4 Myr, cf. Hunter et al. 1995), has been studied extensively, both from the ground and with the HST. A variety of techniques have revealed strong mass segregation, certainly for radii $r \lesssim 0.5$ pc (e.g., Campbell et al. 1992; Larson 1993; Malumuth \& Heap 1994; Hunter et al. 1995; Brandl et al. 1996).

These results suggest that some initial mass segregation already may occur during the formation of the cluster.

\subsection{Disentangling dynamical from primordial effects}

To disentangle the effects of dynamical versus primordial mass segregation, de Grijs et al. (2002c) studied six rich LMC clusters of different ages and masses. All clusters in their sample show clear evidence of mass segregation: (i) the luminosity function slopes steepen with increasing cluster radius, and (ii) the brighter stars are characterized by smaller core radii. More importantly, for all sample clusters, both the slope of the luminosity function in the cluster centres and the degree of mass segregation were found to be similar to each other, within observational errors of a few tenths of power-law slope fits to the data. They conclude that the initial MFs must have been very similar, down to $\sim 0.8-1.0 M_{\odot}$.

Upon closer inspection of these data, however, we do notice significant differences among the degrees of mass segregation among the clusters, which may have been caused by dynamical mass segregation effects (for the older objects), in addition to probable primordial mass segregation. In Fig. 5 of de Grijs et al. (2002c) the behaviour of the slope of the stellar luminosity function as a function of radius (which steepens outward), for different age bins, are found to scale as 1:(0.8 \pm 0.2$):(0.2 \pm 0.1)$ for ages of $10^{7}: 10^{8}: 10^{9} \mathrm{yr}$. This implies that the mass segregation due to dynamical effects becomes significant on time-scales of a few $\times 10^{8}$ yr. Dynamical mass segregation is expected to occur on the half mass relaxation time. Our empirical estimate of the time at which dynamical mass segregation occurs is consistent with, or perhaps relatively short compared to, estimates for the half-mass relaxation time-scales of Galactic GCs (e.g., Gnedin \& Ostriker 1997) which might be caused by differences in the relaxation times.

Combining the results of the studies of very young clusters with the observed changes in the radial dependence of the slope of the mass function with age, we may conclude that (most, if not all) clusters are formed with primordial mass segregation and that the mass segregation increases on a half-mass relaxation time of a few $10^{8} \mathrm{yr}$. This agrees with the results of a recent study of mass segregation in very young open clusters in the solar neighbourhood by Schilbach et al. (2006). In the group of the youngest clusters, with ages between 5 and $30 \mathrm{Myr}$, they found about as many clusters with as without initial mass segregation.

\section{Mass loss from a cluster by stellar evolution and dissolution}

In order to describe the expected effects of changes in the mass function of clusters due to mass segregation and the preferential 
loss of low-mass stars, we first describe the overall mass loss of a cluster by stellar evolution and dissolution.

The loss of mass due to stellar evolution and to dissolution can be described as

$\frac{\mathrm{d} M}{\mathrm{~d} t}=\left(\frac{\mathrm{d} M}{\mathrm{~d} t}\right)_{\mathrm{ev}}+\left(\frac{\mathrm{d} M}{\mathrm{~d} t}\right)_{\mathrm{dis}}$,

The mass loss due to stellar evolution is

$\left(\frac{\mathrm{d} M}{\mathrm{~d} t}\right)_{\mathrm{ev}}=M_{i} \frac{\mathrm{d} \mu_{\mathrm{ev}}(t)}{\mathrm{d} t}=\frac{M}{\mu_{\mathrm{ev}}(t)} \frac{\mathrm{d} \mu_{\mathrm{ev}}(t)}{\mathrm{d} t}$,

where $1-\mu_{\mathrm{ev}}(t)=q_{\mathrm{ev}}(t)$ is defined as the mass fraction of the initial mass that is lost due to stellar evolution. Thus, $M(t)=$ $M_{i} \mu_{\mathrm{ev}}(t)$ if there is no dissolution. The function $q_{\mathrm{ev}}(t)$ can be expressed as the simple approximation

$\log q_{\mathrm{ev}}(t)=\left(\log t-a_{\mathrm{ev}}\right)^{b_{\mathrm{ev}}}+c_{\mathrm{ev}}$ for $t>12.5 \mathrm{Myr}$.

The values of $a_{\mathrm{ev}}, b_{\mathrm{ev}}$ and $c_{\mathrm{ev}}$ were derived by Lamers et al. (2005b) from the GALEV cluster evolution models for different metallicities, $0.0004 \leq Z \leq 0.05$. For solar metallicity they found $a_{\mathrm{ev}}=7.00, b_{\mathrm{ev}}=0.255$ and $c_{\mathrm{ev}}=-1.805$. Equation (3) describes the mass-loss fraction of the models at $t>12.5 \mathrm{Myr}$, with an accuracy of a few per cent. The mass loss at younger ages is negligible because the most massive stars with $M_{*}>30 M_{\odot}$ hardly contribute to the mass of the cluster.

The mass loss due to dissolution can be written as

$\left(\frac{\mathrm{d} M}{\mathrm{~d} t}\right)_{\mathrm{dis}}=-\frac{M}{\tau_{\mathrm{dis}}}=-\frac{M}{t_{0} M^{\gamma}}=-\frac{M^{1-\gamma}}{t_{0}}$,

where the instantaneous dissolution time-scale $\tau_{\text {dis }}$ is assumed to depend as a power law on the mass of the cluster, as $\tau_{\text {dis }}=t_{0} M^{\gamma}$. Boutloukos \& Lamers (2003) and Lamers et al. (2005a) derived the value $\gamma$ empirically from a statistical study of cluster samples in different galaxies and found it to be $0.62 \pm 0.05$. BM2003 derived the same value of $\gamma=0.62$ from their $N$-body simulations of clusters in tidal fields, in excellent agreement with the empirical studies. The constant $t_{0}$ depends on the tidal field at the location of the cluster.

Lamers et al. (2005b) have shown that the numerical solution of Eq. (1) can be approximated to a high degree of accuracy by the analytical expression

$M(t) \simeq M_{i} \times\left\{\left(\mu_{\mathrm{ev}}(t)\right)^{\gamma}-\frac{\gamma t}{t_{0} M_{i}^{\gamma}}\right\}^{1 / \gamma}$,

where $M_{i}$ is the initial mass of the cluster.

Equation (5) is valid if the first term on the right-hand side is greater than the second term. In the opposite situation, i.e., when the mass lost through dissolution is larger than the mass remaining after mass loss via stellar evolution, then $M(t)=0$ and the cluster is completely dissolved. Lamers et al. (2005b) compared this expression for the mass evolution of star clusters with the results of the $N$-body simulations of BM2003, and found that the agreement is extremely good, i.e., within a few per cent over more than $98 \%$ of the lifetime of the clusters.

The total dissolution time of clusters can be derived from Eq. (5). Lamers et al. (2005b) showed that it is approximately

$t_{\mathrm{dis}}\left(M_{i}\right)=6.60 \times 10^{2}\left(\frac{M_{i}}{10^{4}}\right)^{0.653} \times t_{0} 0.967-0.00825 \times \log \left(M_{i} / 10^{4}\right)$

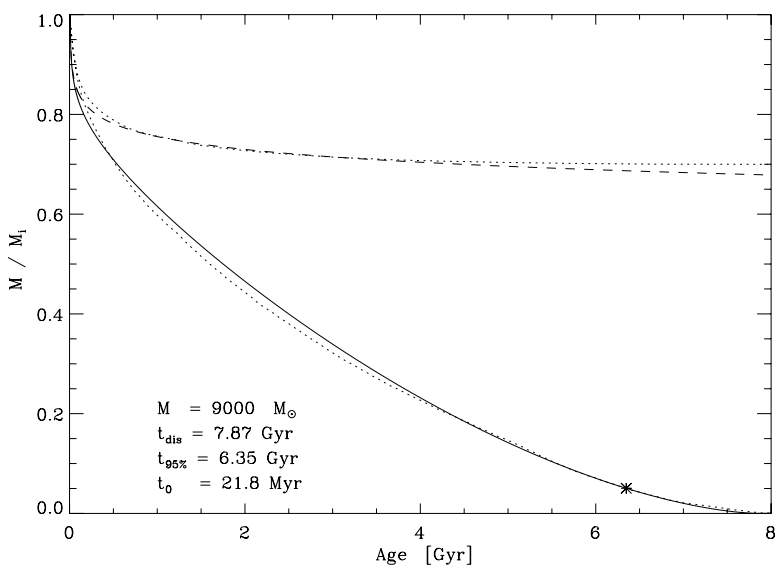

Fig. 1. The decreasing mass of a cluster with an inital mass of $9000 M_{\odot}$, predicted by Eq. (5) with $t_{0}=21.8 \mathrm{Myr}$. The solid line is the decreasing mass due to stellar evolution and dissolution, the dashed line shows the decreasing mass if stellar evolution were the only mass-loss mechanism. This agrees very well with the $N$-body simulations by BM2003 of a cluster of $9000 M_{\odot}$ in a circular orbit at a galactocentric distance of $8.5 \mathrm{kpc}$ with an initial concentration parameter of $W_{0}=5$ (dotted lines). The asterisk indicates the moment when $95 \%$ of the initial mass is lost. This is the value of $t_{\mathrm{dis}}^{\mathrm{BM}}$.

for solar metallicity and $\gamma=0.62$. They also showed that the time when $95 \%$ of the initial mass is lost, which is the dissolution time-scale adopted by BM2003, $t_{\mathrm{dis}}^{\mathrm{BM}}$, is approximately $0.8 t_{\mathrm{dis}}$.

Figure 1 shows the decreasing mass of a cluster with an initial mass of $9000 M_{\odot}$, described by Eq. (5). The value of $t_{0}=21.8 \mathrm{Myr}$ was chosen in such a way that the cluster has lost $95 \%$ of its mass in $6.35 \mathrm{Gyr}$, which is the value derived by BM2003 from $N$-body simulations for a cluster of $9000 M_{\odot}$ with an initial concentration parameter of $W_{0}=5$ in a circular orbit at $R_{\text {gal }}=8.5 \mathrm{kpc}$. The total dissolution time of such a cluster is $t_{\mathrm{dis}}=7.87 \mathrm{Gyr}$, which is $24 \%$ greater than $t_{\mathrm{dis}}^{\mathrm{BM}}$. Fig. 1 shows that Eq. (5) agrees very well with the result from $N$-body simulations.

\section{The changing mass function of a cluster}

In the previous section we described the decreasing mass of the cluster due to evolutionary and dynamical processes. In this section, we describe how stellar mass loss and cluster dissolution change the stellar MF of the cluster during its evolution.

\subsection{The changes in the mass function predicted by $\mathrm{N}$-body simulations}

BM2003 calculated the dynamical evolution of a large grid of clusters in the tidal field of the Galaxy by means of $N$-body simulations. The clusters have different masses and different initial density distributions which are expressed in terms of King profiles with central concentration parameters $W_{0}=5.0$ or 7.0. The clusters orbit at different galactocentric distances, from 2.8 to $15 \mathrm{kpc}$, in circular or elliptical orbits. The initial masses of these cluster models are in the range from 4000 to $70000 M_{\odot}$. An initial Kroupa (2001) MF of the type $N(M) \mathrm{d} M=M^{-\alpha} \mathrm{d} M$ with $\alpha=2.3$ for $0.5 \leq M \leq 100 M_{\odot}$ and $\alpha=1.3$ for $0.1 \leq M<0.5 M_{\odot}$ was adopted. BM2003 defined the dissolution time of their models as the age at which only $5 \%$ of the initial mass remains due to stellar evolution and dissolution. We 
will refer to these dissolution times as $t_{\mathrm{dis}}^{\mathrm{BM}}$. The dissolution times of these models are in the range $2.3<t_{\mathrm{dis}}^{\mathrm{BM}}<40 \mathrm{Gyr}$.

The $N$-body simulations of BM2003 show several remarkable features:

(a) the preferential loss of low-mass stars starts at about the same fractional age of the cluster, i.e. at $t_{\text {segr }} \simeq 0.20 t_{\text {dis }}$

(b) before this age the fractional decrease of the number of stars is almost independent of mass (except for the most massive stars whose number decreases because of stellar evolution). This is because stars of almost all masses can be ejected by encounters with the most massive ones;

(c) after $t_{\text {segr }}$ the cluster mainly loses high-mass stars, due to stellar evolution, and low-mass stars due to evaporation, but almost no stars of intermediate mass;

(d) the changes in the mass function at the low mass end, $M \leq 2 M_{\odot}$, as a function of $t / t_{\text {dis }}$ are very similar for all models, despite large differences in the initial cluster mass, dissolution time and ellipticity of the orbit of the cluster.

\subsection{Simplified models: the concept}

Based on the results of the $N$-body simulations, we can derive a simple model that agrees with the basic results of BM2003 and that allows us to subsequently calculate the photometric evolution of dissolving clusters.

We describe the effects of stellar evolution and cluster dissolution on the stellar MF by the following approximations:

(1) stellar evolution removes stars at the high-mass end of the MF but leaves the rest of the mass distribution unchanged. The stellar upper mass limit decreases with time;

(2) initially, dissolution will remove stars of all masses with about equal probability. This means that the number of stars of all masses will decrease but the slope of the mass distribution will remain unchanged. This agrees with the results of the $N$-body simulations;

(3) when mass segregation has occurred, dissolution preferentially removes low-mass stars from the periphery of the cluster. In our simple model we will assume that in this phase, i.e. at $t>t_{\text {segr }}$, the dynamical effects remove only the stars with the lowest remaining mass from the cluster. Thus, the lower mass limit of the cluster stars will increase to higher values. Stellar evolution will continue to reduce the upper mass limit during all phases. (In fact, the evolution of stars of all masses is fully taken into account in our method because we use the results from the GALEV cluster evolution models.)

(4) the transition between phases (2) and (3) is likely to be gradual. Therefore, we assume that a fraction $1-f_{\text {segr }}$ of the mass that is lost due to dynamical effects is independent of the mass and the fraction $f_{\text {segr }}$ is lost in the form of low-mass stars only. The factor $f_{\text {segr }}$ can be specified as a function of time and increases from some initial value $f_{0} \equiv f_{\text {segr }}(t=0)$ to $f_{\text {segr }}=1$ at $t>t_{\text {segr }}$. If there is no primordial mass segregation then $f_{0}=0$.

These assumptions have the advantage that the slope of the mass distribution remains constant during all phases of a cluster's evolution. Only the maximum and minimum stellar mass (due to effects 1 and 3, respectively), and the constant describing the total number of stars (due to effect 2) change as a function of time. With these simplifications, we can study and understand the expected changes in the photometry of star clusters during their evolution. Figure 2 shows the concept of our approximations.

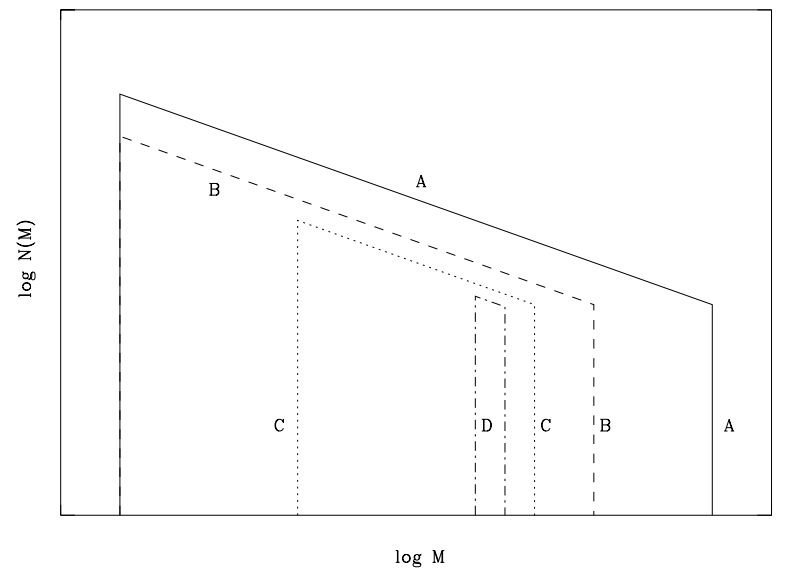

Fig. 2. Schematic changes in the stellar MF, $\log (N)$ versus $\log (M)$, of a cluster due to stellar evolution and dissolution. The initial MF is assumed to be a power law. A: the inital MF. B: Stellar evolution has removed the most massive stars and dynamical effects, before clusterwide mass segregation, have reduced the number of stars of all masses with equal probability. C: after mass segregation has occurred dynamical effects have removed the lowest-mass stars. D: the MF just before the cluster is completely dissolved.

In this simple description the MF gets narrower with time and reaches a single mass just before the cluster is fully dissolved.

Our assumptions are in agreement with those of the $N$-body simulations of the dynamical evolution of clusters in tidal fields by BM2003 (see Sect. 4.1). The main difference is in the shape of the mass function at the low-mass end. BM2003 showed that the MF at $M<2 M_{\odot}$ becomes steeper with time, i.e. $\mathrm{d} \log (N) / \mathrm{d} \log (M)$ becomes more positive after core collapse. In our model we approximate this by a MF that keeps its original slope but with a lower mass limit that shifts to higher $M$ as time progresses. The total amount of mass at $M<2 M_{\odot}$ is the same in both the BM2003 models and in our models.

\subsection{Changes in the mass function due to stellar evolution}

We adopt a power-law stellar IMF, $N(M) \mathrm{d} M=C(t) M^{-\alpha} \mathrm{d} M$ with $\alpha=2.35$, i.e., a Salpeter IMF, in the range of $M_{\min }(t)<M<$ $M_{\max }(t)$, with $M_{\min }(0)=0.15$ and $M_{\max }(0)=70 M_{\odot}$. With this value of $M_{\min }(0)$ the mean stellar mass in a cluster is very similar to that of the Kroupa (2001) MF. The factor $C(t)$ is related to the luminous mass of the cluster at time $t$ via

$M_{l}(t)=\frac{C(t)}{\alpha-2}\left\{M_{\min }(t)^{2-\alpha}-M_{\max }(t)^{2-\alpha}\right\}$.

Stellar evolution reduces the mass of the cluster because of the evolution of its most massive stars. We will assume that massive stars keep their initial mass until they reach the end of their life. This is a reasonable assumption because the dominant mass loss for our study occurs after about $10 \mathrm{Myr}$, when stars with $M>12 M_{\odot}$ no longer exist. Stars with $M<12 M_{\odot}$ have no appreciable mass loss during the main-sequence phase, and so their mass loss occurs in the relatively short time of less than about $10 \%$ of the main-sequence lifetime at the end of their life. For $M_{\max }(t)$ we adopt the main-sequence turn-off lifetime of stars given by Hurley et al. (2000).

\subsection{Changes in the mass function due to dissolution}

If a fraction $1-f_{\text {segr }}$ of the mass lost by dissolution is independent of the stellar mass and the fraction $f_{\text {segr }}$ is lost in the form of 

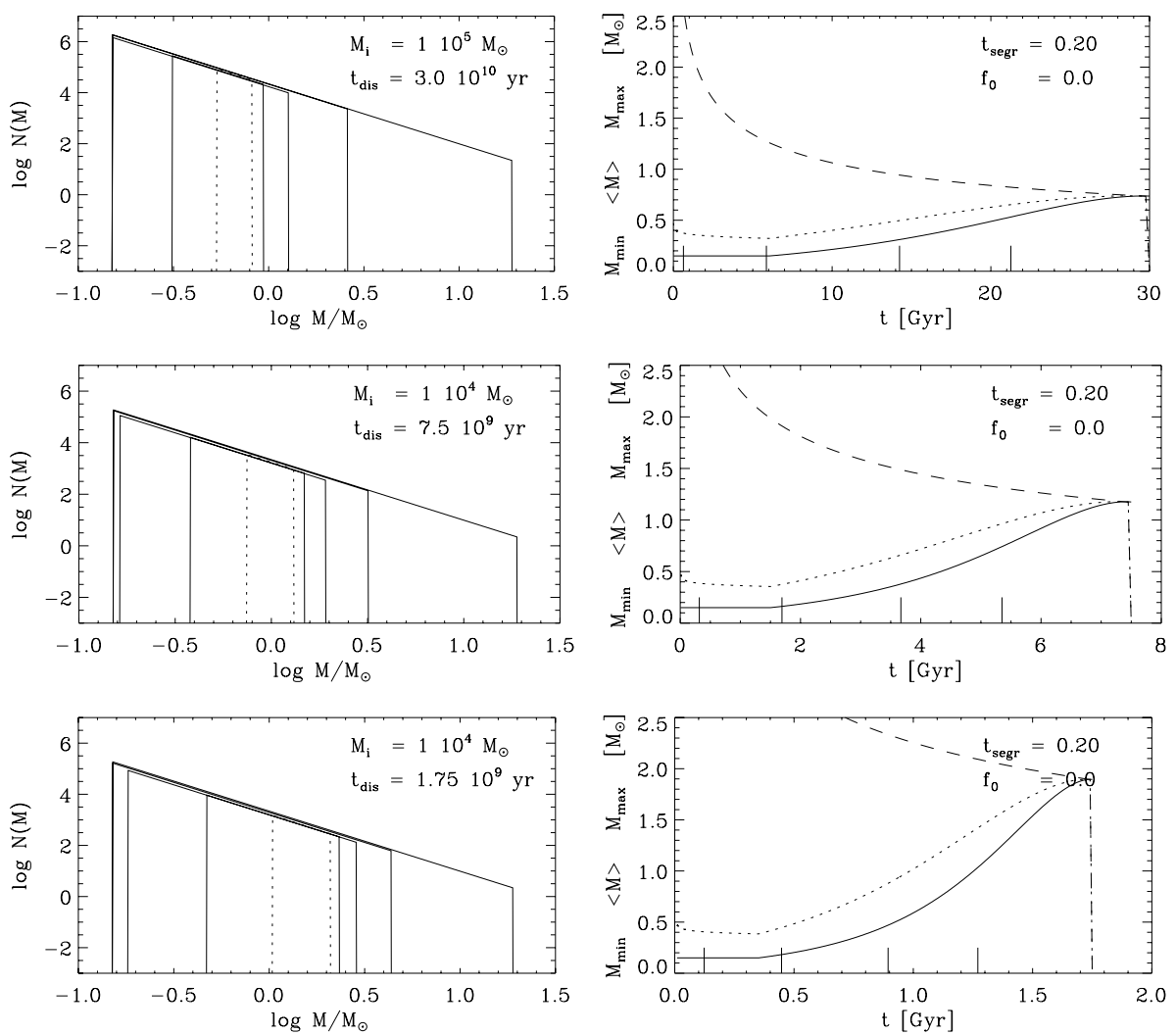

Fig. 3. The changes in the stellar mass function (excluding remnants) of dissolving clusters with a total dissolution time of $t_{\text {dis }}=30,7.5$ and $1.75 \mathrm{Gyr}$ (from top to bottom). The preferential loss of low-mass stars sets in at $t_{\mathrm{segr}}=0.20 t_{\mathrm{dis}}$. The left-hand panels show the mass function when the mass of the cluster is 100, 75, 50, 25 and 10\% of its initial mass $M_{i}$ (from outer to inner envelope). The dotted line shows the MF when $M(t)=0.10 M_{i}$. The right-hand panels show the changes in the maximum (dashed line), minimum (solid line) and mean (dotted line) stellar mass as a function of time for the same models. The vertical tick marks indicate the times when the mass of the cluster is reduced to $75,50,25$ and $10 \%$ of $M_{i}$ respectively (from left to right).

the lowest-mass stars, then we can find an expression for the increase of the stellar minimum mass with time by combining Eqs. (4) and (7). This yields

$$
\begin{aligned}
\frac{\mathrm{d} M_{\min }^{2-\alpha}}{\mathrm{d} t} & =\frac{(\alpha-2) f_{\mathrm{segr}}}{C(t)}\left(\frac{\mathrm{d} M}{\mathrm{~d} t}\right)_{\mathrm{dis}} \\
& =\frac{(\alpha-2) f_{\mathrm{segr}}}{C(t) t_{0}} M^{1-\gamma} .
\end{aligned}
$$

Similarly, by combining Eq. (4) with the derivative of Eq. (7) we find an expression for the decrease of the constant $C(t)$ of the mass function,

$\frac{\mathrm{d} \ln C(t)}{\mathrm{d} t}=\left(1-f_{\text {segr }}\right)\left(\frac{\mathrm{d} \ln M}{\mathrm{~d} t}\right)_{\mathrm{dis}}=\frac{1-f_{\text {segr }}}{t_{0}} M^{-\gamma}$.

For any time-dependent segregation parameter, $f_{\text {segr }}(t)$, we can calculate the evolution of the MF of a cluster by solving Eqs. (7)-(9).

\subsection{Simple models}

To demonstrate the effect of mass segregation and the preferential loss of low-mass stars on the time-dependent MF we assume that $f_{\text {segr }}$ increases in time as a step function (in agreement with the results of the $N$-body simulations).
$f_{\text {segr }}=0$
if $t<t_{\text {segr }}$
$f_{\text {segr }}=1$
if $t \geq t_{\text {segr }}$

where $t_{\text {segr }}$ is the time when cluster-wide mass segregation has occurred. The models of BM2003 suggest that $t_{\text {segr }} \simeq 0.20 t_{\text {dis }}$ if there is no initial mass segregation. However, observations suggest that clusters may be born with initial mass segregation, in which case $t_{\text {segr }}=0$. We consider models with $t_{\text {segr }}=0$ and $t_{\text {segr }}=0.2 t_{\text {dis }}$.

Figure 3 shows the changes in the stellar mass function (without the remnants) of clusters with different dissolution times between 1.75 and 30 Gyr. The longer the dissolution time, the smaller the mass of the most massive stars at the end of the cluster's life. The lower-mass limit starts shifting to higher masses only after $t_{\text {segr }}$, which is assumed to be $0.20 t_{\text {dis }}$ in these models. The mean stellar mass at the end of the cluster's life is equal to the mass at the main-sequence turn-off at $t=t_{\mathrm{dis}}$. By that time, the lower-mass limit has increased to the same value. The mean mass first decreases from its initial value of $0.545 M_{\odot}$ due to the rapid loss of the massive stars to about $0.35 M_{\odot}$, and increases after $t_{\text {segr }}$ due to the preferential loss of low-mass stars. The mean mass at the end of the cluster's lifetime is 0.75 , 1.2 and $2.9 M_{\odot}$ for dissolution times of $30,7.5$ and $1.75 \mathrm{Gyr}$, respectively.

The mass functions are independent of the adopted initial cluster mass, and only depend on $t_{\text {dis }}$ and $t_{\text {segr. }}$. The evolution of the mean mass for the model with $t_{\text {dis }}=30 \mathrm{Gyr}$ resembles that predicted by BM2003 for a cluster of $7.1 \times 10^{4} M_{\odot}$ with $t_{\mathrm{dis}}^{\mathrm{BM}}=23.7 \mathrm{Gyr}$ and $t_{\mathrm{dis}}=30.2 \mathrm{Gyr}(\mathrm{BM} 2003$, Fig. 15). The difference is significant mainly near the end of the cluster's life, after $24 \mathrm{Gyr}$, where BM2003 predict a rapid increase in the mean mass to $1.35 M_{\odot}$ compared to our value of $0.75 M_{\odot}$. This is 

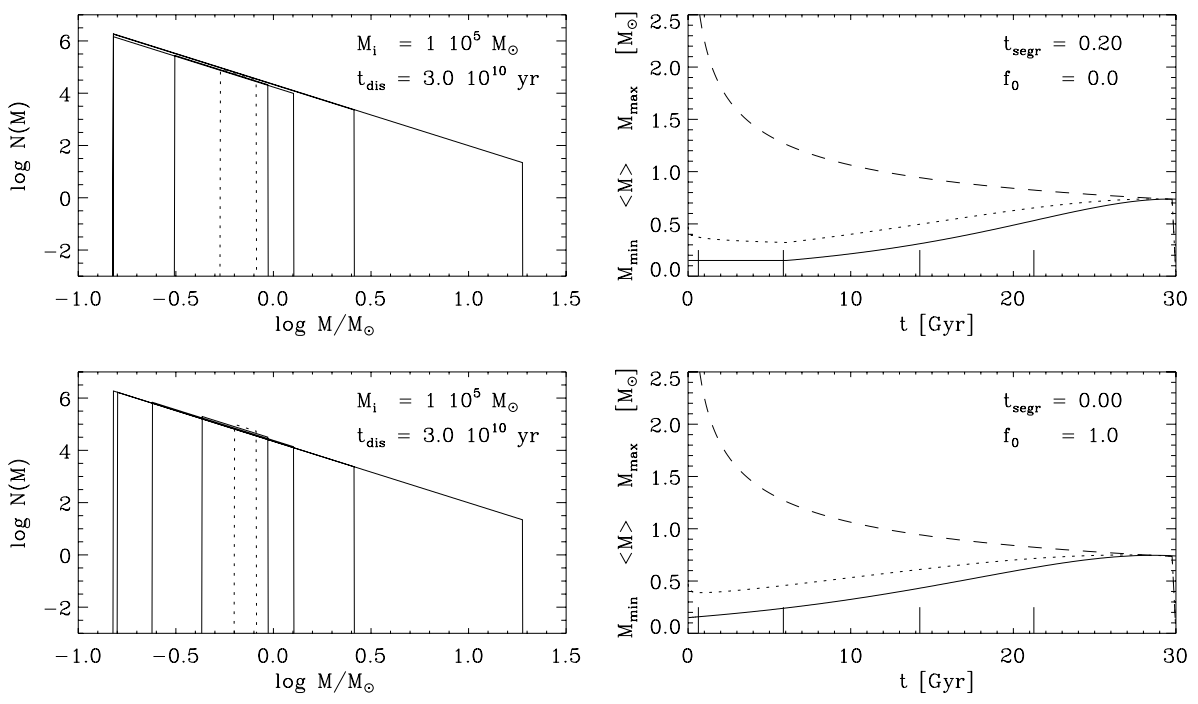

Fig. 4. The changes in the mass function of a cluster with and without initial mass segregation (top and bottom, respectively), plotted in the same way as in Fig. 3. Notice the difference in the mass function at the low-mass end.

because the mass function calculated by BM2003 also includes the remnants of the massive stars, mainly neutron stars of about $1.5 M_{\odot}$. Our mass function is for luminous stars only.

Figure 4 shows the effect of initial mass segregation on the evolution of the stellar mass function of clusters. The top panel is for $t_{\text {segr }}=0.20 t_{\text {dis }}$ and the lower panel is for $t_{\text {segr }}=0$, i.e. for initial mass segregation. The difference results in a different evolution of the mass function at the low-mass end. In the case of initial mass segregation, the mean mass hardly decreases during the early phases, because the rapid loss of high-mass stars due to stellar evolution is more than compensated for by the simultaneous loss of low-mass stars.

\section{The photometric history of dissolving clusters}

In this section we calculate the photometric history of dissolving star clusters based on our description of the changes in the mass function. We adopt the GALEV models with a Salpeter IMF of exponent -2.35 . The change in the upper mass limit due to stellar evolution is included in the GALEV models. The changes in the lower mass limit are described in Sect. 4. The GALEV models are based on the stellar evolution calculations of the Padova group and include, amongst others, a description of the AGB evolution. We will see that these AGB stars are important for the colours of old clusters.

We calculated models with and without initial mass segregation $\left(t_{\text {segr }}=0.0\right.$ and 0.2 respectively) in Eq. (10)), and with total dissolution times of $0.1,0.3,1,3,10$ and 30 Gyr. The results are shown in Fig. 5 for two models with $t_{\text {dis }}=1 \mathrm{Gyr}$ (left) and 10 Gyr (right).

The upper panels of Fig. 5 show the changes in the mass and visual magnitude of a cluster with an initial mass of $10^{5} M_{\odot}$. The lower panels show the changes in the photometric history in $B-V, V-I$ and $V-K$. The solid lines show the prediction including the loss of low-mass stars but without initial segregation $\left(f_{\text {segr }}=0, t_{\text {segr }}=0.2\right)$. The dotted lines show the results of the standard GALEV models, i.e. without the preferential loss of low-mass stars. We note that the colours are independent of the adopted initial mass of the cluster, but $M_{V}$ has to be scaled to the decreasing cluster mass.

Figure 5 shows that near the end of the cluster's life the visual luminosity becomes fainter than in case of no preferential loss of low-mass stars. This is because of the missing contribution from the large number of lost low-mass main-sequence stars.

The colour evolution of models with preferential loss of lowmass stars is different to that of the standard models. At ages between about $0.4<t / t_{\text {dis }}<0.8$, clusters that include mass segregation become slightly bluer than clusters without mass segregation (lower panels of Fig. 5). This effect is stronger for clusters with increasing values of $t_{\text {dis }}$. This means that it will be stronger for more massive clusters, which have a longer lifetime, than for low-mass clusters. This is due to the loss of red low-mass mainsequence stars. The situation changes drastically after $t \simeq 0.8 t_{\text {dis }}$ when the clusters with mass segregation become much redder than those without mass segregation. This is due to the fact that late in the lifetime of a cluster its red colour is dominated by AGB stars. Stars at the low-mass end of the main sequence are bluer than AGB stars, so the loss of stars at the low-mass end of the main sequence makes the cluster redder. The blueing of the clusters at $0.4 \leq t / t_{\mathrm{dis}} \leq 0.8$ implies that the age of clusters derived from standard cluster evolution models will be underestimated, whereas the age will be overestimated for clusters with $t \geq 0.8 t_{\text {dis. }}$

The key question is whether we can distinguish this effect on the basis of the location of clusters in colour-colour diagrams. Figure 6 shows the evolution of the dissolving clusters in two colour-colour plots of $B-V$ vs. $V-I$ and $V-K$ vs. $V-I$ for the same two models as shown in Fig. 5, i.e. with $t_{\text {dis }}=1$ and $10 \mathrm{Gyr}$, respectively. The reddening of the clusters after $t>0.8 t_{\mathrm{dis}}$ is highlighted by marking the colours at $t=0.80,0.90$ and $0.95 t_{\text {dis }}$. The difference is $\Delta(V-I)=0.3$ and 0.2 mag for the models with $t_{\text {dis }}=1$ and $10 \mathrm{Gyr}$, respectively.

Notice that in all colour-colour plots the reddening of the clusters due to the loss of low-mass stars occurs almost along the lines of the normal colour-colour history (dashed lines in Fig. 6). This implies that the effect of the preferential loss of low-mass stars cannot easily be distinguished on the basis of colour-colour plots. Hence, it could lead to errors in the age determination of unresolved clusters.

Since clusters with mass segregation evolve almost along the same lines as the standard cluster models in colour-colour diagrams, we can plot the expected error in the age determination versus one of the colours. The size of this error in the age 

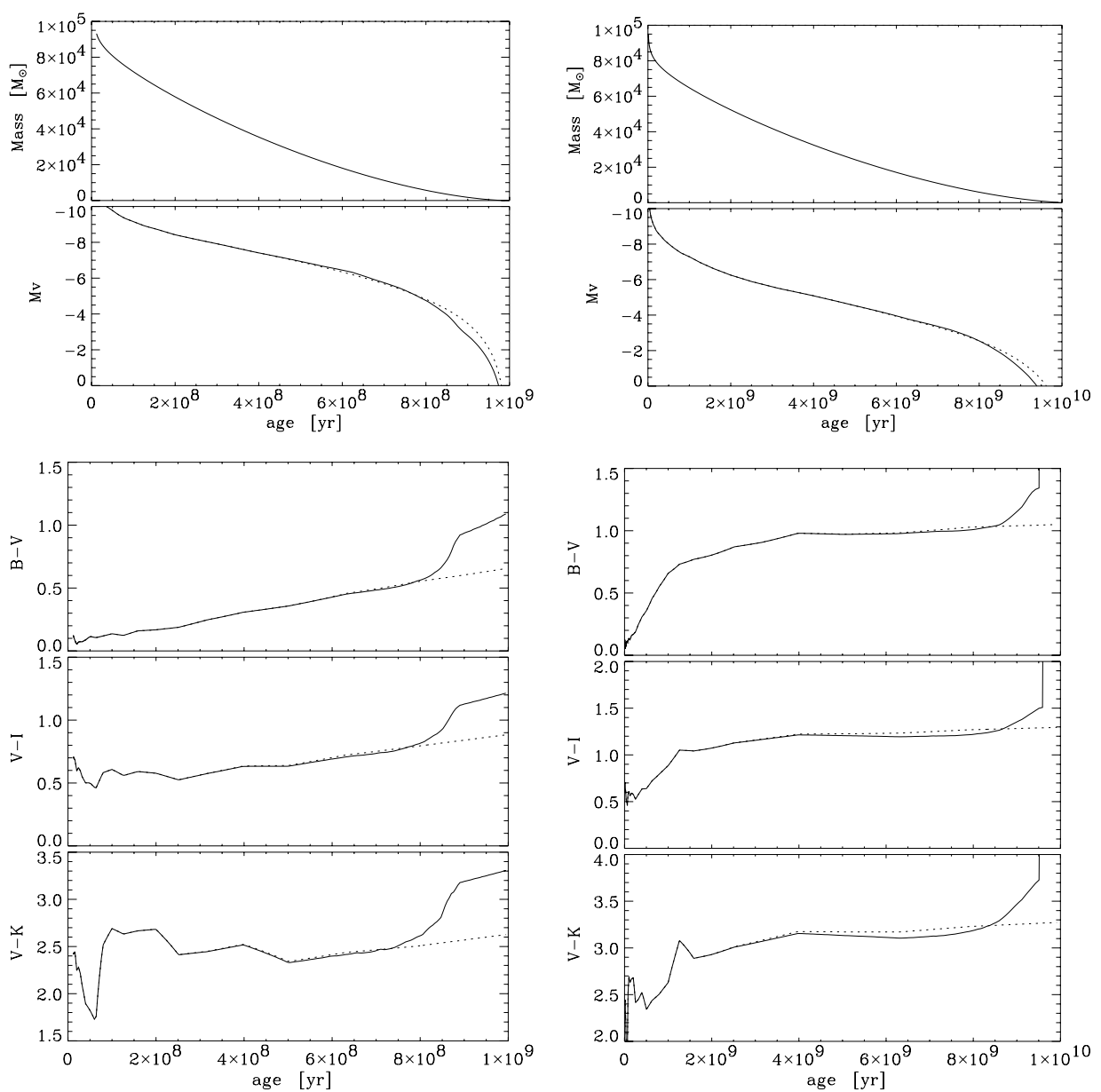

Fig. 5. The mass, $M_{V}$, and colour history of dissolving star clusters with mass segregation and the preferential loss of low-mass stars taken into account. Left-hand figure: a cluster with an initial mass of $10^{5} M_{\odot}$, an initial Salpeter stellar IMF, no initial mass segregation and a dissolution time of 1 Gyr. The right-hand figure is for a similar model but with $t_{\mathrm{dis}}=10 \mathrm{Gyr}$. The upper panels show the changes in mass and $M_{V}$ and the lower panels the changes in HST/WFPC2 broad-band colours. The solid lines show the photometric history of dissolving clusters with the preferential loss of low-mass stars, as described in Sect. 4. The dashed lines show the photometry of dissolving clusters without the preferential loss of low-mass stars (i.e. the standard model).

estimate is shown in Fig. 7. In the upper panel we plot the age of a cluster versus its $V-I$ colour. The solid line shows the relation for the GALEV cluster evolution models without mass segregation. The dashed lines show the predicted colour-age relations for clusters where mass segregation results in the preferential loss of low-mass stars. Notice that the age estimated on the basis of standard cluster models without mass segregation will be roughly correct for the first $40 \%$ of the cluster's lifetime, i.e. for $\log (t)<\log \left(0.4 t_{\text {dis }}\right)=\log \left(t_{\text {dis }}\right)-0.4$. After that time a cluster with mass segregation first gets slightly bluer than its counterpart without mass segregation, so the age will be underestimated when conventional cluster evolution models are used. This correction is about $0.15 \mathrm{dex}$ in $\log (t)$ for clusters with $t_{\mathrm{dis}} \simeq 3 \mathrm{Gyr}$, $0.30 \mathrm{dex}$ for $t_{\mathrm{dis}} \simeq 10 \mathrm{Gyr}$ and $0.5 \mathrm{dex}$ for $t_{\mathrm{dis}} \simeq 30 \mathrm{Gyr}$ (lower panel of Fig. 7). This situation changes during the last $20 \%$ of the lifetime of the cluster when mass segregation and the preferential loss of low-mass stars make the cluster much redder than in conventional models. During that time, the cluster age will be largely overestimated when cluster models without mass segregation are used to derive the age from the colours. The age correction during that time reaches values as large as -0.6 dex.

We conclude that the ages of clusters derived from the broadband $B, V, I$ and $K$ colours using standard cluster evolution models may be affected by significant systematic errors. During the first $40 \%$ of the lifetime of a cluster its age will be approximately correct. During $0.4<t / t_{\mathrm{dis}}<0.8$ the age will be underestimated. During the last $20 \%$ of the cluster's lifetime the age will be overestimated by a factor that rapidly increases towards the end of its lifetime.

(We have also calculated the photometric history of clusters with primordial mass segregation, i.e. with $t_{\text {segr }}=0$, but these models are very similar to those without initial mass segregation. If there is no primordial mass segregation, this will be established within a short fraction, $\sim 20 \%$, of the cluster's lifetime.)

\section{Discussion}

We have studied the effect of mass segregation and the preferential loss of low-mass stars on the photometric evolution of unresolved star clusters. The following assumptions were made:

(a) We adopted the GALEV cluster evolution models, which are based on the Padova evolutionary tracks that include a desription of the AGB evolution. This is important because stars in the AGB phase are the main contributors to the colour and brightness in long-wavelength bands after about 0.1 Gyr. We adopted the models for solar metallicity and for a Salpeter IMF with index -2.35 down to an initial 

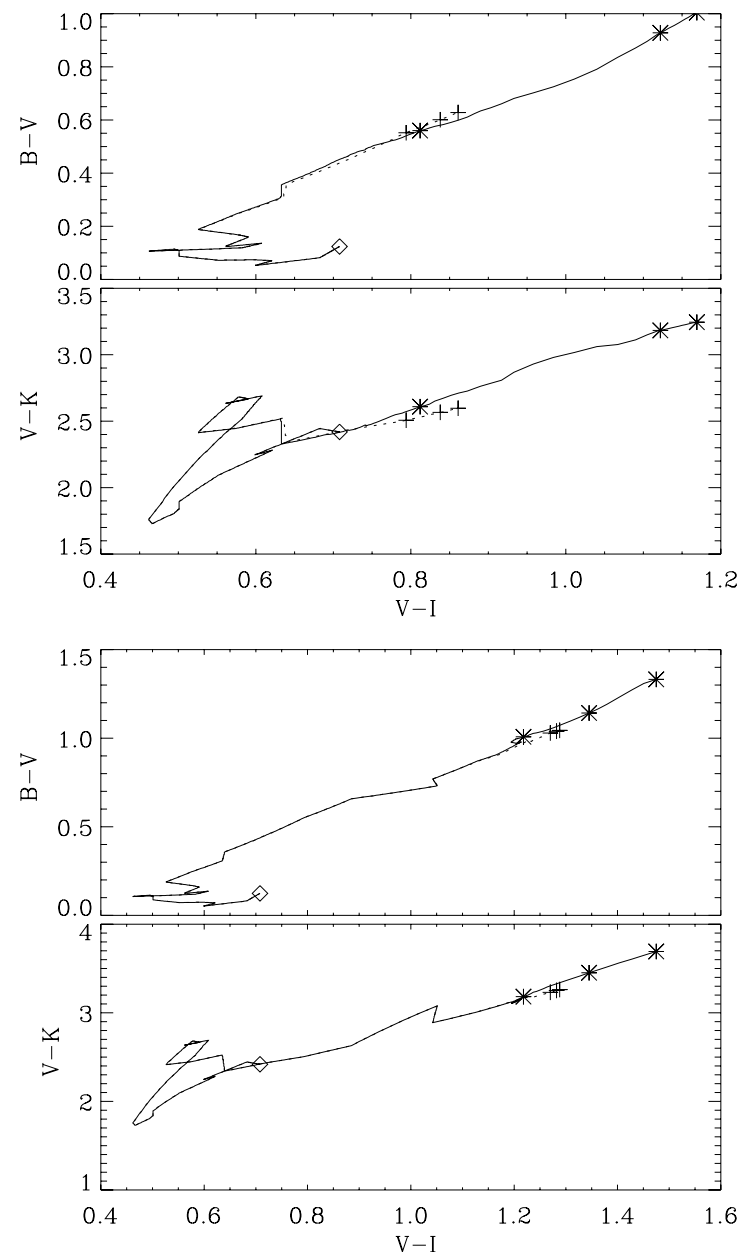

Fig. 6. The evolution of dissolving star clusters in the colour-colour diagram of the HST/WFPC2 broad-band filters $B-V$ vs. $V-I$ and $V-K$ vs. $V-I$, for clusters without initial mass segregation $\left(f_{\text {segr }}=0.0\right.$ and $t_{\text {segr }}=0.2$ ). Upper panel: $t_{\mathrm{dis}}=1 \mathrm{Gyr}$; lower panel: $t_{\mathrm{dis}}=10 \mathrm{Gyr}$. The solid line shows the colour-colour evolution with mass segregation and the preferential loss of low-mass stars and the dashed lines show the evolution without the loss of low-mass stars, i.e. the evolution of the GALEV models. The diamond shows the colours in the beginning, at $t=12 \mathrm{Myr}$. The three asterisks show the colours of the cluster at three different ages: $t / t_{\mathrm{dis}}=0.80,0.90$ and 0.95 (reddest point). The three crosses show the colours of the standard GALEV models at the same time. Notice that the colours get much redder near the end of the lifetime of the clusters.

lower-mass limit of $M_{\min }=0.15 M_{\odot}$. Mass loss due to stellar evolution is fully taken into account in our models.

(b) We simulated the effect of the preferential loss of low-mass stars due to mass segregation in a simple way, by gradually removing the lowest-mass stars. This is a simplification of the expected changes in the stellar IMF due to the preferential loss of low-mass stars (see Fig. 7 of BM2003). This is not a bad approximation, because we have tuned the increasing low-mass limit to the results of the $N$-body simulations in such a way that our model has the correct loss of lowmass stars due to evaporation. We have shown that it results in a predicted evolution of the mean stellar mass that is very similar to that of the $N$-body simulations of BM2003. Since the mean stellar mass after about $0.1 \mathrm{Gyr}$ (i.e. after the early high-mass loss phase of massive stars) is mainly dependent on the cluster content of low-mass stars, the agreement

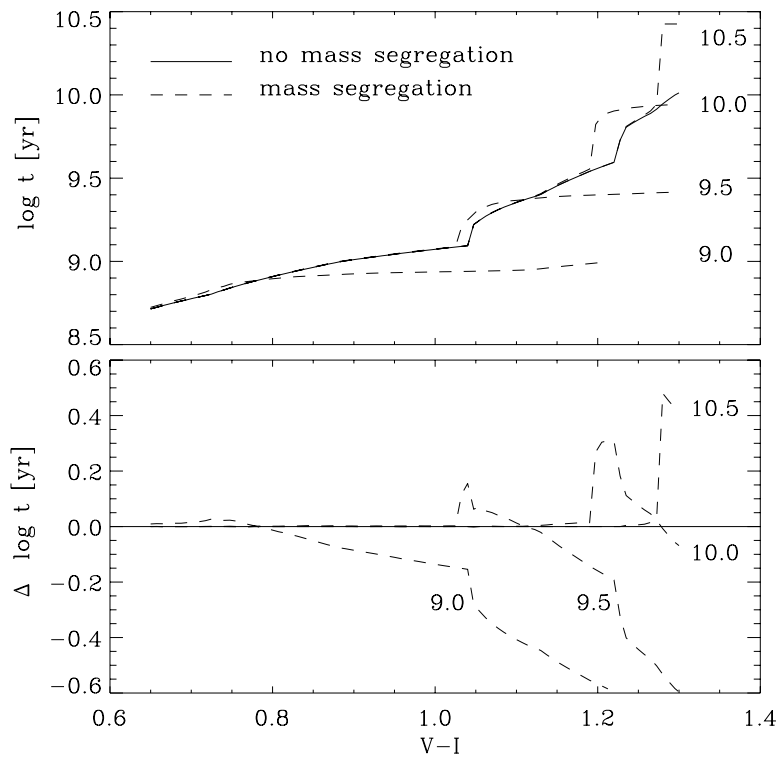

Fig. 7. Upper panel: the relation between $V-I$ and the corresponding age of a cluster. The solid line is for clusters without mass segregation. The dashed lines are for clusters with mass segregation and the preferential loss of low-mass stars, for different dissolution times. The parameters $\log \left(t_{\mathrm{dis}}\right)$ are indicated in the panel. Lower panel: the logarithmic age correction to be made to ages derived from standard cluster evolution models.

between our simplified model and the $N$-body simulations supports the accuracy of our predictions.

(c) This first study is only for clusters with solar metallicity. The same predicted effects can be expected qualitatively for lower-metallicity clusters. In a follow-up study we will extend the calculations to a large range of metallicities (Lamers $\&$ Anders, in prep.).

(d) N-body simulations of BM2003 without initial mass segregation show that cluster-wide mass segregation occurs after about $20 \%$ of the total lifetime of a cluster. On the other hand, observations of very young clusters in the solar neighbourhood (Schilbach et al. 2005) and in the Magellanic Clouds (de Grijs et al. 2002c; and Sect. 2) show that a fraction of the clusters are born with initial mass segregation, i.e. with the massive stars concentrated towards the center and the lower-mass stars in the periphery. Therefore, we have done two sets of calculations, one with initial mass segregation and one with mass segregation after $20 \%$ of the total lifetime of the cluster.

(e) In our descriptive model, the cluster loses stars of all masses proportional to its mass function in the initial phase at $t<$ $t_{\text {segr }}$ (i.e. before mass segregation has occurred). This means that the slope of the IMF does not change during this phase. After mass segregation has been established only the lowestmass stars are ejected from the cluster in our model. This results in a gradual shift of the lower stellar mass limit. During all phases, stellar evolution removes the most massive stars. These two effects result in a narrowing of the mass function. In our model the upper mass limit and the lower mass limit meet at the moment the cluster completely dissolves. This is close, but not identical, to the predicted changes in the mass function in the $N$-body simulations by BM2003.

(f) We assume that stars lost from the cluster do no longer contribute to the photometry of unresolved clusters. The validity of this assumption depends on the spatial resolution of the photometry, i.e. on the point spread function (PSF) of the 
observations of unresolved clusters, and on the speed with which stars leave the cluster. As a simple estimate we assume that the stars leave the cluster typically with a velocity of the order of the escape velocity. In that case it is easy to show that the time it takes for a star to move beyond the radius of the PSF is $1 \times 10^{7} r_{\mathrm{PSF}} \sqrt{R_{\mathrm{cl}} / M_{\mathrm{cl}}} \mathrm{yr}$, when $r_{\mathrm{PSF}}$ and $R_{\mathrm{cl}}$ are the radius of the PSF and the cluster, respectively, in pc and $M_{\mathrm{cl}}$ is the cluster mass in $M_{\odot}$, if the path of the star is perpendicular to the line of sight. For average directions this time will be about $4 / \pi$ as long. For a typical cluster of $10^{4} M_{\odot}$ and $R_{\mathrm{cl}}=3 \mathrm{pc}$ an ejected star will leave the PSF with a radius of $1 \mathrm{pc}$ (corresponding to $0.04^{\prime \prime}$ at $d=5 \mathrm{Mpc}$ ) in about $2 \times 10^{5}$ yr. Therefore, unless the PSF covers a large area of many $\mathrm{pc}^{2}$, our assumption is reasonable.

With these assumptions we predicted the photometric evolution of unresolved star clusters and found that in the age range of $0.4 \lesssim t / t_{\text {dis }} \lesssim 0.8$ the cluster will be bluer than predicted by standard cluster evolution models. In the age range of $t \gtrsim 0.8 t_{\mathrm{dis}}$ the clusters will be redder than predicted by standard models.

The discovery of a group of apparent intermediate-age (2-5 Gyr old) globular clusters in the giant elliptical galaxy NGC 4365 by Forbes (1996), Gebhardt \& Kissler-Patig (1999) and Larsen et al. (2001) may be the result of mass segregation and the preferential loss of low-mass stars. The luminosityweighted age of clusters in NGC 4365 is about 14 Gyr and there is no evidence of a recent merger. Another puzzling effect is the lack of field stars in the age range from 2-5 Gyr. The clusters were reanalysed by Brodie et al. (2005) and Larsen et al. (2005). They confirm the intermediate age based on the broadband colours. However, when Lick indices are used to derive the ages of these clusters, the ages are between 10 and 14 Gyr, as expected for this galaxy. Interestingly, the apparent intermediateage clusters are concentrated more towards the center of the galaxy than the other clusters.

We suggest that the apparent intermediate age clusters in NGC 4365 may be old clusters, 13 Gyr, that have lost a significant fraction of their low-mass stars due to mass segregation and tidal stripping. If this is the correct explanation, then the age of the clusters is now between about 40 and $80 \%$ of their total lifetime, which implies that their total lifetime is expected to be about 16 to 32 Gyr. These are reasonable values for the dissolution times of massive clusters (BM2003). The concentration of these clusters towards the center of the galaxy might be explained by the fact that the total cluster lifetime, $t_{\mathrm{dis}}$, is shorter close to the centers of galaxies than far from the center. BM2003 have shown that for galaxies with a logarithmic gravitational potential the dissolution time scales linearly with the distance to the galactic center (except for interacting galaxies). The clusters in NGC 4365 that do not show this apparent intermediate age are, on average, at greater distances from the galactic center, so they have longer total lifetimes. Their present age of $13 \mathrm{Gyr}$ is too small to already show the effects of mass segregation.

In this explanation we did not discuss the effect of metallicity, which also plays a role both in stellar mass loss and in the colour evolution, because the present study was done for clusters with solar metallicity only. In our follow-up study (Lamers $\&$ Anders, in prep.) we will predict the effects of mass segregation and the preferential loss of low-mass stars for a large range of metallicities.

\section{Conclusions}

We predicted the photometric evolution of unresolved clusters with different total life times, $t_{\mathrm{dis}}$, in the $B, V, R, I$ and $K$ bands of the HST/WFPC2 broad-band filter system, with mass loss due to stellar evolution and evaporation of low mass stars taken into account. The photometric evolution is compared with that of cluster models not including mass segregation or the preferential loss of low-mass stars. We call these the "standard" models. We considered clusters with a total lifetime in the range of 0.3 to 30 Gyr. We obtained the following results.

1. During the first part of the lifetime of the cluster, i.e. during the first $\sim 40 \%$, irrespective of the total life time $t_{\text {dis }}$, the photometric evolution is the same as predicted for the standard models if the decreasing mass is taken into account. The dissolution of the cluster makes it fainter in all bands, but the colours are unaffected.

2. Between $\sim 40$ and $\sim 80 \%$ of its total lifetime the cluster is bluer than predicted by the standard models. This is due to the fact that the cluster has lost a large fraction of its (red) low-mass stars. This effect is small: $\Delta(V-I) \simeq 0.03$ mag for clusters with $t_{\text {dis }}=1 \mathrm{Gyr}$, but increases steeply with increasing $t_{\mathrm{dis}}$. It is about $0.1 \mathrm{mag}$ for $t_{\mathrm{dis}}=10 \mathrm{Gyr}$. This implies that the age of the clusters will be underestimated when standard models are used. The error in the age estimate is about $0.15 \mathrm{dex}$ in $\log (t)$ if $t_{\mathrm{dis}}=3 \mathrm{Gyr}, 0.30 \mathrm{dex}$ if $t_{\text {dis }}=10 \mathrm{Gyr}$ and 0.5 dex if $t_{\text {dis }}=30$ Gyr. Thus, the age of clusters with a total lifetime of $20 \mathrm{Gyr}$ and a real age of $14 \mathrm{Gyr}$ will erroneously be estimated as about $4 \mathrm{Gyr}$ on the basis of the $V-I$ and $V-K$ photometry.

3. Between about $\sim 0.80$ and $1.0 t_{\text {dis }}$ the clusters are much redder than predicted by the standard models. At those ages the AGB stars are the dominant contributors to the photometry at long wavelengths. These AGB stars are redder than the stars at the low-mass end of the main sequence. The removal of the lowest mass main-sequence stars will make the cluster redder than predicted by standard models. This effect increases from $\Delta(V-I) \simeq 0.0 \mathrm{mag}$ at $t \simeq 0.8 t_{\text {dis }}$ to $0.3 \mathrm{mag}$ at $0.95 t_{\text {dis }}$. This reddening will result in an overestimate of the age of clusters based on broad-band photometry from $B-V$ to $V-K$ colours if standard cluster evolution models are used. The effect is large and can grow to an overestimate of a factor $\sim 4$ near the end of the cluster's life.

4. The changes in colour due to mass segregation and the preferential loss of low-mass stars occurs almost along the same lines in the colour-colour plots as the photometric evolution of the standard models. This makes it difficult to distinguish this effect from reddening due to the age of clusters.

5. The predicted photometric history of clusters with initial cluster-wide mass segregation is indistinguishable from that of clusters without initial mass segregation. This is due to the fact that mass segregation will occur quite rapidly (within $\sim 0.20 t_{\text {dis }}$ ), even if there were no initial mass segregation. Because both the total lifetime of a cluster and the time for mass segregation depend on the half-mass relaxation time, mass segregation will occur at about a constant fraction of $t_{\text {dis. }}$.

$\mathrm{N}$-body simulations have shown that mass segregation and the preferential loss of low-mass stars will occur in clusters in tidal fields. Even if there is no initial mass segregation, the effects predicted by our models will occur in real clusters. 
Acknowledgements. We thank the International Space Science Institute (ISSI) in Bern, Switzerland, for hosting three star cluster workshops, in 2004 and 2005, when this project was carried out. We thank Mark Gieles, Marcel Haas, Diederik Kruijssen and Simon Portegies Zwart for comments and suggestions.

\section{References}

Anders, P., \& Fritze-v. Alvensleben, U. 2003, A\&A, 401, 1063 (GALEV models) Baumgardt, H., \& Makino, J. 2003, MNRAS, 340, 227 (BM2003)

Bonnell, I. A., \& Davies, M. B. 1998, MNRAS, 295, 691

Boutloukos, S. G., \& Lamers, H. J. G. L. M. 2003, MNRAS, 338, 717

Brandl, B., Sams, B. J., Bertoldi, F., et al. 1996, ApJ, 466, 254

Brandl, B., Brandner, W., Eisenhauer, F., et al. 2001, in Extragalactic Star Clusters, ed. E. K. Grebel, \& D. Geisler (ASP: San Francisco), IAU Symp., 207, 226

Brodie, J. P., Strader, J., Denicoló, G., et al. 2005, AJ, 129, 2643

Bruzual, G. A., \& Charlot, S. 1993, ApJ, 405, 538

Campbell, B., et al. 1992, AJ, 104, 1721

de Grijs, R., Johnson, R. A., Gilmore, G. F., \& Frayn, C. M. 2002a, MNRAS, 331, 228

de Grijs, R., Gilmore, G. F., Johnson, R. A., \& Mackey, A. D. 2002b, MNRAS, 331, 245

de Grijs, R., Gilmore, G. F., Mackey, A. D., et al. 2002c, MNRAS, 337, 597

Elson, R. A. W., Tanvir, N., Gilmore, G. F., Johnson, R. A., \& Beaulieu, S. F. 1999, in New Views of the Magellanic Clouds, ed. Y.-H. Chu, N. Suntzeff, J. Hesser, \& D. Bohlender (Canada: Victoria), IAU Symp., 190, 417

Fischer, P., Pryor, C., Murray, S., Mateo, M., \& Richtler, T. 1998, AJ, 115, 592

Forbes, D. A. 1996, AJ, 112, 954

Gebhardt, K., \& Kissler-Patig, M. 1999, AJ, 118, 1526

Gnedin, O. Y., \& Ostriker, J. P. 1997, ApJ, 474, 223

Grebel, E. K. 2004, in The Formation and Evolution of Massive Young Star Clusters, ed H. J. G. L. M. Lamers, L. J. Smith, \& A. Nota (ASP: San Francisco), ASP Conf. Ser, 322,101

Gouliermis, D., Keller, S. C., Kontizas, M., Kontizas, E., \& Bellas-Velidis, I. 2004, A\&A, 416,137
Hillenbrand, L. A. 1997, AJ, 113, 1733

Hillenbrand, L. A., \& Carpenter, J. M. 2000, ApJ, 540, 236 Hillenbrand, L. A., \& Hartmann, L. E. 1998, ApJ, 492, 540 Hoogerwerf, R., de Bruijne, J. H. J., \& de Zeeuw, P. T. 2000, ApJ, 544, L133

Hunter, D. A., Shaya, E. J., Holtzman, J. A., et al. 1995, ApJ, 448, 179

Hurley, J. R., Pols, O. R., \& Tout, C. A. 2000, MNRAS, 315, 543

Inagaki, S., \& Saslaw, W. C. 1985, ApJ, 292, 339

Kontizas, M., Hatzidimitriou, D., Bellas-Velidis, I., et al. 1998, A\&A, 336, 503

Kroupa, P. 2001, MNRAS, 322, 231

Lamers, H. J. G. L. M., Gieles, M., \& Portegies Zwart, S. F. 2005a, A\&A, 429, 173

Lamers, H. J. G. L. M., Gieles, M., Bastian, N., et al. 2005b, A\&A, 441, 117

Larsen, S. S., Brodie, J. P., Huchra, J. P., Forbes, D. A., \& Grillmaier, C. 2001, AJ, 121, 2974

Larsen, S. S., Brodie, J. P., \& Starder, J. 2005, A\&A, 443, 413

Leitherer, C., Schaerer, D., Goldader, J. D., et al. 1999, ApJS, 123, 3

Larson, R. B. 1993, in The Globular Cluster-Galaxy Connection, ed. G. H. Smith, \& J. P. Brodie, ASP Conf. Ser., 48 (ASP: San Francisco), 675

Malumuth, E. M., \& Heap, S. R. 1994, AJ, 107, 1054

Nürnberger, D. E. A., \& Petr-Gotzens, M. G. 2002, A\&A, 382, 537

Portegies Zwart, S. F., Makino, J., McMillan, S. L. W., \& Hut, P. 1999, A\&A, 348, 117

Salpeter, E. 1995, ApJ, 121, 161

Santiago, B. X., Beaulieu, S., Johnson, R., \& Gilmore, G. F. 2001, A\&A, 369, 74

Schilbach, E., Kharchenko, N. V., Piskunov, A. E., Röser, S., \& Scholz, R.-D. 2006, A\&A, in press

Schulz, J., Fritze-v. Alvensleben, U., Möller, C. S., \& Fricke, K. J. 2002, A\&A, 392, 1 (GALEV models)

Sirianni, M., Nota, A., De Marchi, G., Leitherer, C., \& Clampin, M. 2002, ApJ, 579, 275

Stolte, A., Brandner, W., Brandl, B., Zinnecker, H., \& Grebel, E. K. 2004, AJ, 128, 765

Sung, H., \& Bessell, M. S. 2004, AJ, 127, 1014

Testi, L., Palla, F., Prusti, T., Natta, A., \& Maltagliati, S. 1997, A\&A, 320, 159

Westerlund, B. E. 1961, Uppsala Astr. Obs. Ann., 5(1) 